\title{
Integrative Physiology in Modelica
}

\author{
Jiř́i Kofránek ${ }^{1}$, Tomáš Kulhánek ${ }^{1}$, Marek Mateják ${ }^{1}$, Filip Ježek ${ }^{1}$, Jan Šilar ${ }^{1}$ \\ ${ }^{1}$ Department of Pathophysiology, 1st Faculty of Medicine, Charles University \\ \{kofranek, tmkulhanek, matejak.marek, jezekf, janeksilar\}@gmail.com
}

\begin{abstract}
The integrative model of human physiology connects individual physiological subsystems into a single unit. They are enormous (contain thousands of variables) and represent a formalized description of interconnected physiological regulations. The issue of formalization of physiological systems became part of a series of international projects (e.g. the worldwide program "PHYSIOME", or the European program "VIRTUAL PHYSIOLOGICAL HUMAN"). The development of large-scale models of human physiology was facilitated by a new generation (i.e. equation-based) of simulation environments, especially by the Modelica language. These models can be used to explain the causal relations of the pathogenesis of many diseases. They can be applied in the evaluation of clinical trials and they can also be used in the core of sophisticated medical simulators.
\end{abstract}

\section{Keywords: Simulation, Physiology, Integrative models}

\section{Introduction}

In 1972, Arthur Guyton published an article (A. C. Guyton, Coleman, and Granger 1972) in the journal Annual Review of Physiology, whose form quite surpassed the usual forms of physiological articles of those times at the very first sight. An extensive diagram, slightly resembling a complex electronic circuit, enclosed as an attachment, was used as introduction, showing interconnection of essential subsystems, that have an effect on circulation, by means of special symbols expressing mathematical operations (addition, subtraction, multiplication, division, integration and functional dependencies). The connections of the elements thus represented mathematical equations (Fig. 1) and the entire scheme provided a visual graphic representation of a set of equations describing the then available ideas of bloodstream regulation including essential connections with other physiological systems of the human organism (Fig. 2). This graphic scheme of Guyton was thus one of the first mathematical descriptions of mutually connected physiological systems in the human organism and it initiated development of physiological research, today sometimes described as integrative physiology.

\section{Integrative physiology modelling}

Modelling is closely related to formalization - i.e. replacement of verbal description of physiological systems with the exact language of mathematics in the form of a mathematical model. Given the complexity of biological systems, the process of formalization is delayed in biological and medical sciences compared to physics, chemistry or technical sciences.

While in physics, the formalization process began at some point during the 17 th century, in medical and biological sciences it occurred only with the onset of cybernetics and computer science. Computer models developed based on mathematical description of biological reality are used as the methodological tool in latter sciences.

In physiology, formalized descriptions have been used since the 1940s; at that time, McCulloch and Pitts

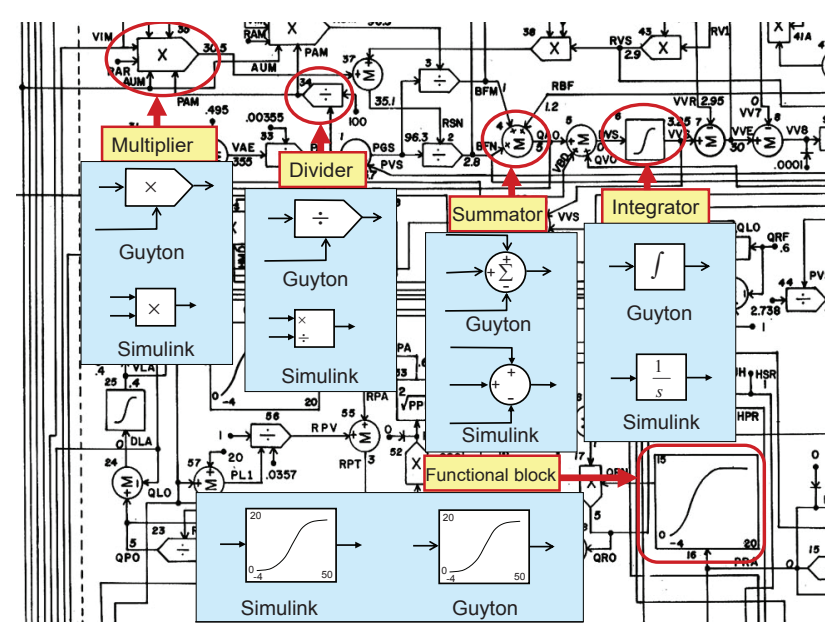

Figure 1. Individual elements in the scheme of Guyton's model display mathematical operations whose connections represent graphically expressed mathematical equations. Blocks in the original Guyton notation (1972), and the same blocks in Simulink (1990).

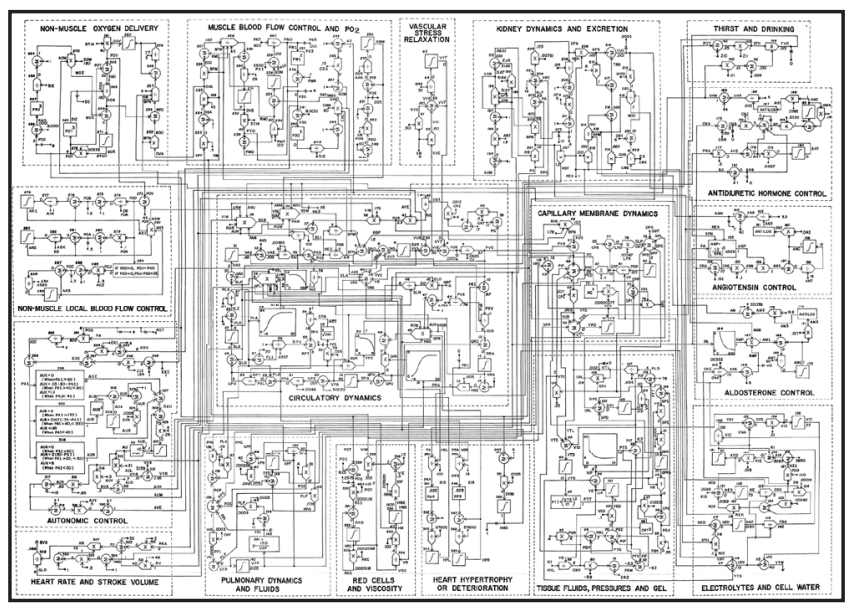

Figure 2. Interconnected physiological subsystems in the Guyton model (Guyton et al., 1972). 
(McCulloch and Pitts 1943; Pitts and McCulloch 1947), to mention some examples, designed a simplified neuron model and Sheppard (Sheppard 1948) introduced his compartment approach, that found quick application in pharmacokinetics. In the 1950s, Hodkgin and Huxley (Hodgkin and Huxley 1952) published their groundbreaking model of the neuronal excitation membrane. In the 1960s, the development of computers supported another wave of publications related to formalized description of physiological reality, for example, Milhorn's monograph on the use of Automated Control Theory in physiological systems (Milhorn 1966) or the pioneering work of Grodins regarding the modelling of respiration (Grodins, Buell, and Bart 1967). At the end of the 1960s and at the beginning of the 1970s of the past century, multicompartment systems found broad application in biology and medicine (Atkins 1969), and computer-based methods were developed to determine the parameters of biological systems (Potůček et al. 1977).

\subsection{Integrative physiology}

The above mentioned model of Guyton and his collaborators from 1972 (A. C. Guyton, Coleman, and Granger 1972) was one of the first extensive mathematical descriptions of physiological functions of mutually connected subsystems in the human organism, which established an area of physiological research, today described as "integrative physiology" (Coleman and Summers 1997). Similarly as theoretical physics seeks to describe physical reality and explain the results of experimental research using formal means, "integrative physiology" also seeks to create a formalized description of mutual connections among physiological regulation systems and to explain their role in the development of various diseases based on experimental results. From this point of view, Guyton's model was a certain milestone aimed at capturing the dynamics of relationships among the regulation systems of the cardiovascular circulation, kidneys, respiration, volume and ion composition of body fluids using a graphically depicted network while applying a system view of physiological regulation systems.

Guyton's graphic notation of the formalized description of physiological relationships, inspired by then commonly used analogue computers, provides a highly visual representation of mathematical correlations - blocks at the network nodes represent graphic symbols for individual mathematical operations, while the connecting lines represent individual variables. Guyton's graphic notation was soon adopted also by other authors - for example, Ikeda et al. (Ikeda et al. 1979) in Japan or the Amosov research group in Kiev (Amosov et al. 1977).

With his research and teaching, Guyton changed physiology from a science of verbal descriptions to one of quantitative analysis. He brought mathematics and physics into the discipline. He was a pioneer in the use of computers to study of body function and has taught scientists all over the world computer simulation.

Guyton's model also served as an inspiration and a resource for the development of complex models of physiological regulation systems used to explain causal chains of reactions in the human organism to various stimuli and to understand the development of various pathological conditions. Among others, the modified Guyton model became part of the foundations for an extensive model of physiological functions in the NASA program "Digital Astronauts" (White and McPhee 2007).

Currently, the international project PHYSIOME (http://www.physiome.org) is focused on the formalized description of physiological systems; this project is the successor of the "GENOME" project, which resulted in a detailed description of the human genome. The aim of the "PHYSIOME" project is to develop a formalized description of physiological functions. Computer models are used as the methodical tool (Bassingthwaighte 2000; P. Hunter, Robbins, and Noble 2002; P. J. Hunter et al. 2006; Peter J. Hunter, Crampin, and Nielsen 2008; Omholt and Hunter 2016). "VIRTUAL PHYSIOLOGICAL HUMAN" (http://www.vph-institute.org) is a European initiative in this field, focused, among other things, on applications of the formalized approach to human physiology in clinical medicine (P. Hunter 2016). Descendants of Guyton's original computer model of the cardiovascular system are some of the resources for the development of present complex models of physiological regulation systems in
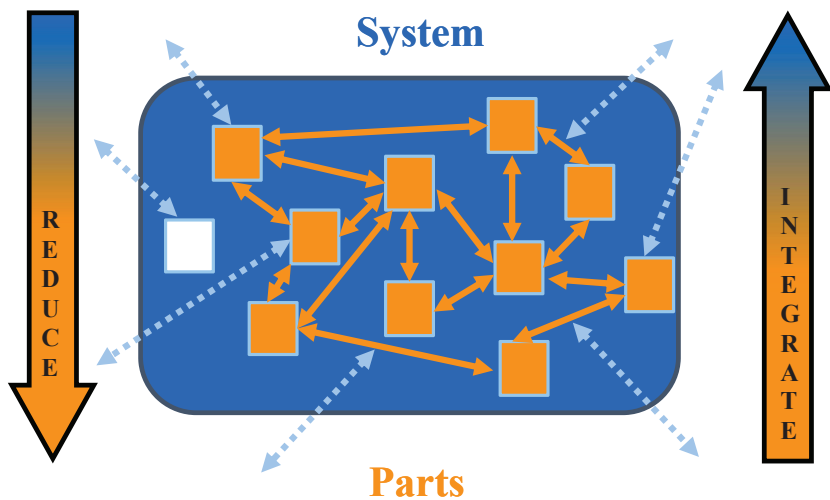

Figure 3. A system as an entity that maintains its existence through mutual interactions of its individual parts (system elements). In the system analysis, the system should include only those elements that enter in mutual interactions with each other (orange squares), while elements that may be structurally and functionally similar to other system elements but interact only with the surroundings of the system (an empty square) should be excluded from the system. The surroundings of the system interact with individual system elements or modulate their mutual bonds (dashed arrows). When studying a system (the transfer between individual hierarchical levels), reductionist and integration tools and methods need to be combined. Adapted from (Peter Kohl and Noble 2009). 
this European project (Thomas et al. 2008).

Besides integrative models of human physiology, integrative models of laboratory animals have also been developed recently. The project "VIRTUAL RAT" is aimed at designing a complex model of a laboratory rat that can be validated more easily by comparing to experimental data from laboratory animals (http://www. virtualrat.org).

\subsection{The human organism as a hierarchical system}

The task of exploring a living organism as a system unit poses a key problem of how (with respect to the explored problem) the system structure should be defined in the biological object, what parts should be understood as system elements, how to define the subsystems, etc.

According to the definition of Bertalanffy (Von Bertalanffy 1973), the system is an entity that maintains its existence through mutual interactions of its individual parts (system elements). Therefore in the system analysis, a system defined on any given real object should include only those parts that primarily interact with each other (see Fig. 3).

System research must include (Peter Kohl and Noble 2009):

- Identification of individual parts of the entity;

- Detailed characteristics of mutually interacting parts of the entity to be included among the system elements (while parts interacting only with the surroundings of the system will not be included);

- Exploration and subsequent description of mutual interactions among individual elements;

- Exploration and subsequent description of interactions with the surroundings of the system (the system surroundings have direct or indirect effects on the system elements, by influencing mutual interactions among the system elements);

- Combinations of reductionist and integration tools and methods in exploration of any system entity on various hierarchical levels.

A system as a set of elements and integration bonds is thus defined on a real object. Based on more detailed exploration of the system entity, an ever a more complex system can be defined, which may be composed of a number of mutually integrating subsystems. However, this is not a purely mechanical process. When passing to a more detailed level, a number of included functions and bonds of the higher hierarchical level must be reduced, and on the contrary, when passing to a higher level a number of elements and bonds must be integrated (Fig. 4). Every model is a simplified notion of representation of reality on various hierarchical levels.
The approach of classical molecular biology goes "from below upwards". It starts from "bottom elements" of the organisms - genes and proteins. Molecular biology models provide formalized descriptions of interactions of gene and protein cell structures that can be used to understand their functions.

The approach of classical physiology is the opposite "from above downwards", somewhat resembling reverse engineering. First, the system is studied on higher levels and subsequently, the process goes down in an effort to find inverse solutions. The system behaviour is used to try to derive the functions of its individual parts.

Integrative models start "from the middle". They combine both approaches - downward to the cellular and molecular level and upward to integration and deriving of functionalities of the human organism as a whole (P. Kohl et al. 2010).

The circulatory system model of the Japanese authors Shim et al. (Shim et al. 2006) can be given as an example of connecting models of various hierarchical levels; these authors combined a simple model of cardiovascular haemodynamics of the vascular system with the model of ventricles of the heart (Fig. 5), acting as a heart pump. The ventricles were modelled, in a simplified manner, as spherical elastic compartments with variable tension of their wall. This tension was obtained from the model of actomyosin cross-bridges of the myocyte (formation of these bridges determines the strength of the stretched

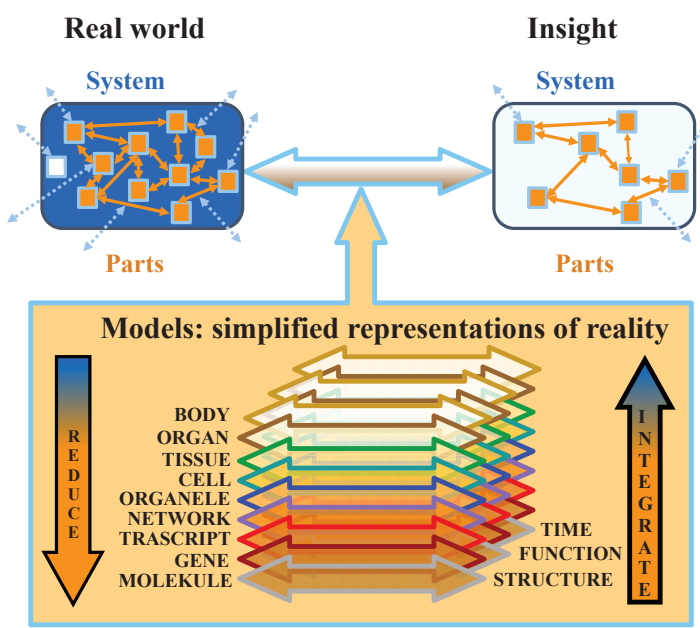

Figure 4. Our understanding of the "real world system" usually provides only a simplified representation of reality. The gradual evolution of our understanding of the real biological world is based on the use and analysis of experimental and theoretical (mathematical) models on all hierarchical levels. The result is reflected in ever more detailed knowledge of the structure of functional relationships and their changes in time, gradually integrated in higher hierarchical levels. System biology provides a framework for targeted interconnection of various aspects of applications of models in biomedical research and development. Adapted from (Peter Kohl and Noble 2009). 


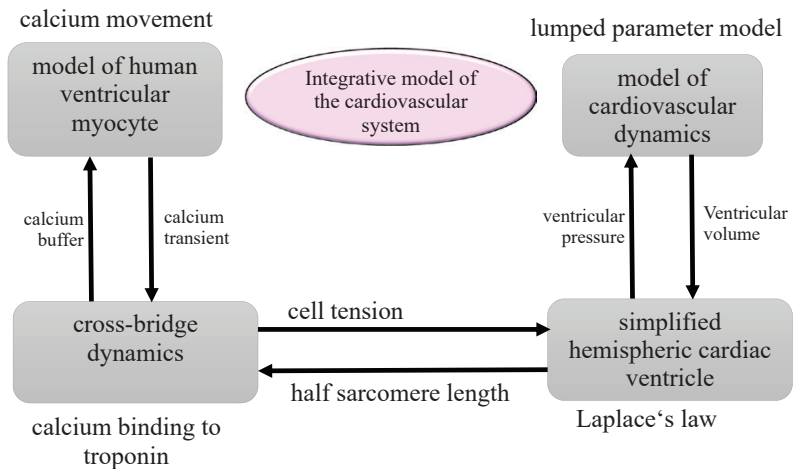

Figure 5. The integrative model of the cardiovascular system as a combination of models on various hierarchical levels according to (Shim et al. 2006).

muscle cell). Their formation is affected by calcium crossing the cell membrane and the sarcoplasmic reticulum membrane where calcium is cyclically released and uptaken. In the sarcoplasmic bridge model, calcium binds to troponin. This binding of calcium causes actomyosin cross-bridges to form, resulting in subsequent tension of the muscle cell. The model of actomyosin cross-bridges was therefore connected to the model of calcium passing between the cytoplasm and sarcoplasmic reticulum.

The cardiovascular haemodynamics model is based on a considerable simplification of reality; it is designed as an RLC model with lumped parameters. Ventricular pressure is found at the input; it is generated by the cardiac ventricular model depicted as a sphere with the wall of variable rigidity. The tension value of the myocyte muscle fibre, being the output of the actomyosin crossbridges (validated according to experimental results), is the starting value for calculating the ventricular wall rigidity. The actomyosin cross-bridge model depends on the output of the myocyte calcium dynamics model (validated according to experimental results). The connection of models of different hierarchical levels integrates important outputs of lower hierarchical level models (for example, behaviour of the myocardium as a whole is derived from the actomyosin cross-bridge model of one cell). Although the models of every hierarchical level represent considerable simplification of reality, the model outputs indicate, for example, the effect of calcium levels in the muscle cell cytoplasm on pressurevolume curves of the left ventricle, thus illustrating, e.g., the clinically verified effect of pharmaceuticals acting on the potassium pump in myocytes.

\subsection{Hummod}

Today, the most extensive model of integrated physiological systems of human physiology is apparently the HumMod model created based on international cooperation of a group of collaborators and disciples of A. Guyton, at the Mississippi University Medical Center, USA (R. L. Hester et al. 2011; R. Hester et al. 2011; Lerant

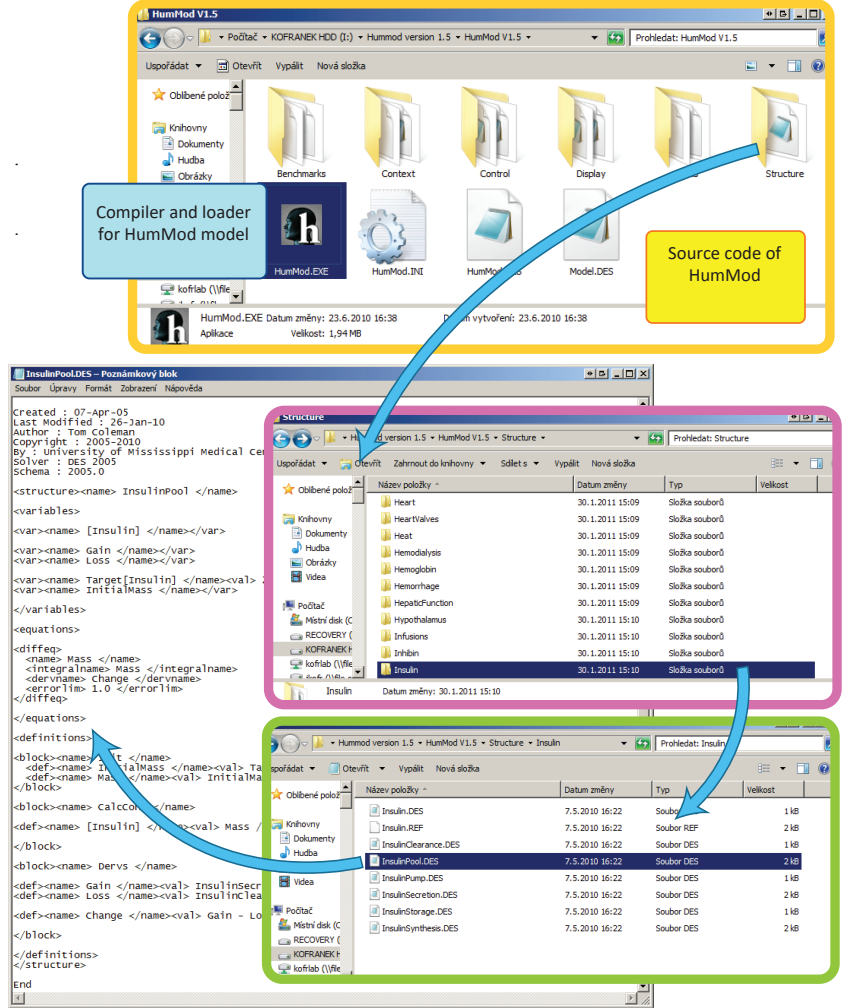

Figure 6. The HumMod simulator has been distributed with a compiler, loader and the source code written in thousands of XML files.

et al. 2015; W. A. Pruett, Clemmer, and Hester 2016).

The authors do not seek to keep its structure a secret; the source text of the model (containing more than 5,000 variables) can be downloaded from the website of the model, http://hummod.org. The source text was written in the special markup language XML. The entire mathematical model is offered as "open source"; users can use the website to download and install on their computer the source code as well as the compiler and run the model on their own machine (Figs. 6 and 7). Users can thus adapt and modify the model. The problem is that the XML source texts of the entire model are written in several thousands of files located in hundreds of folders, and it is very difficult to orient oneself in the mathematical relationships by studying more than a thousand of mutually connected XML files.

In the development of models in the field of integrative physiology, many research teams actually preferred to use older models of complex physiological regulations for example, the old models of Guyton (A. C. Guyton, Coleman, and Granger 1972; A. C. Guyton et al. 1986; A. C. Guyton, Hall, and Montani 1988; J. P. Montani, Mizelle, et al. 1989; J. P. Montani, Adair, et al. 1989; J.-P. Montani and Van Vliet 2009) or the old model of Ikeda (Ikeda et al. 1979). For example, this path was taken by the international research team of the SAPHIR (System Approach for Physiological Integration of Renal, cardiac and respiratory control) project in 2008 after deciding that 


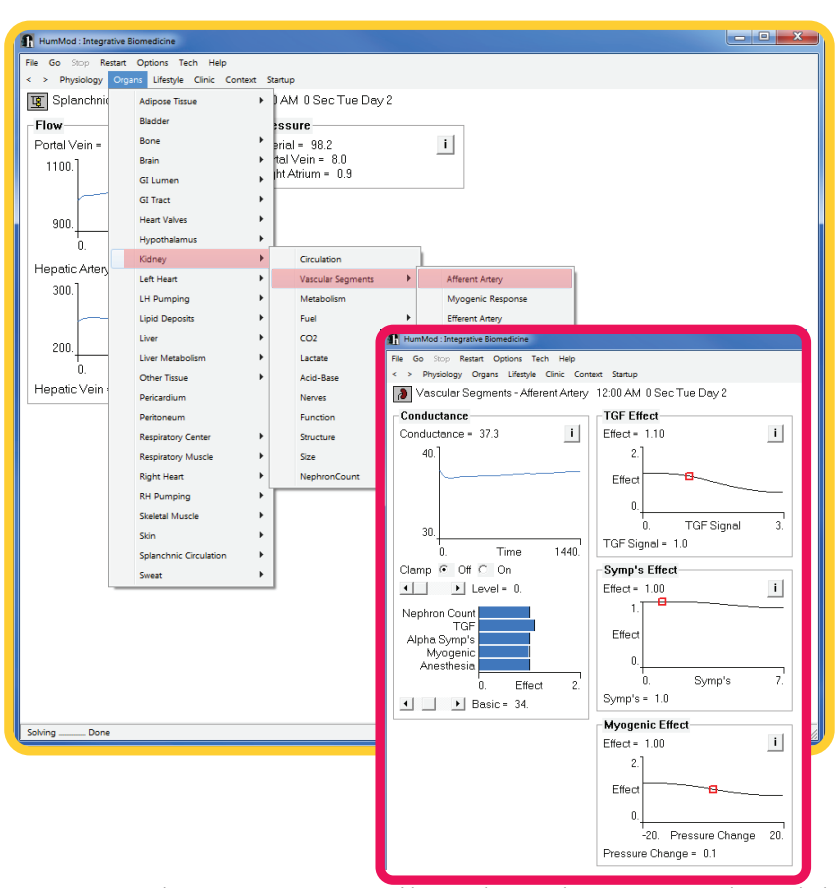

Figure 7. The user can compile and run the HumMod model. Using a widely branched menu, hundreds of variables can be monitored during simulation experiments.

the open source mathematical model of integrative human physiology containing over 3000 variables from Guyton's laboratory (R. L. Hester, Coleman, and Summers 2008) at that time seemed very poorly readable and difficult to understand to the project participants. Therefore two legacy integrated models have served as a starting point for integrative model development (Thomas et al. 2008), namely the classic model of Guyton et al. (1972), which focused on blood pressure regulation, and the model of Ikeda et al. (1979), based on Guyton's models but extended to focus on the overall regulation of body fluid. The model of Ikeda was recently reimplemented in the modern simulation environment (Fontecave-Jallon and Thomas 2015). Similarly, in 2011 Mangourova et al. (Mangourova, Ringwood, and Van Vliet 2011) implemented in Simulink an older model of Guyton of 1992 written in C instead of the then most recent (but difficult to understand for them) version of the large integrative model - $\boldsymbol{Q H P}$ (Quantitative Human Physiology - the predecessor of the HumMod model) from Guyton's laboratory.

It is apparent that comprehensibility of descriptions of complex integrative models is one of their limiting factors for their acceptance by the scientific community. If the creators are the only ones to understand their models, their potential of factual communication with other scientists is thus hindered. This also limits the possibilities of designing integrative models within a broader scientific community. This is an important reason why the development of methodologies has been gaining importance; such methodologies would make the descriptions of structures of complex hierarchical models clearer in a way so that a wider spectrum of users can understand them.

Special viewers have been created for better understanding of the HumMod model that enable the user to go through individual relationships within the model (Xu et al. 2011; Wu et al. 2013; Chen et al. 2013). In spite of this., the equations of the model and their relationships still remain difficult to understand for the user.

\subsection{Our results - Modelica libraries and PHYSIOMODEL}

One of the ways to facilitate the understanding of complex hierarchical models consists in using the new object-oriented modelling language Modelica. Therefore we have decided to reimplement the entire complex model of the American authors in this language.

We were not frightened by the complex structure of the HumMod model (called QHP in the previous version) and established closer cooperation with the American authors.

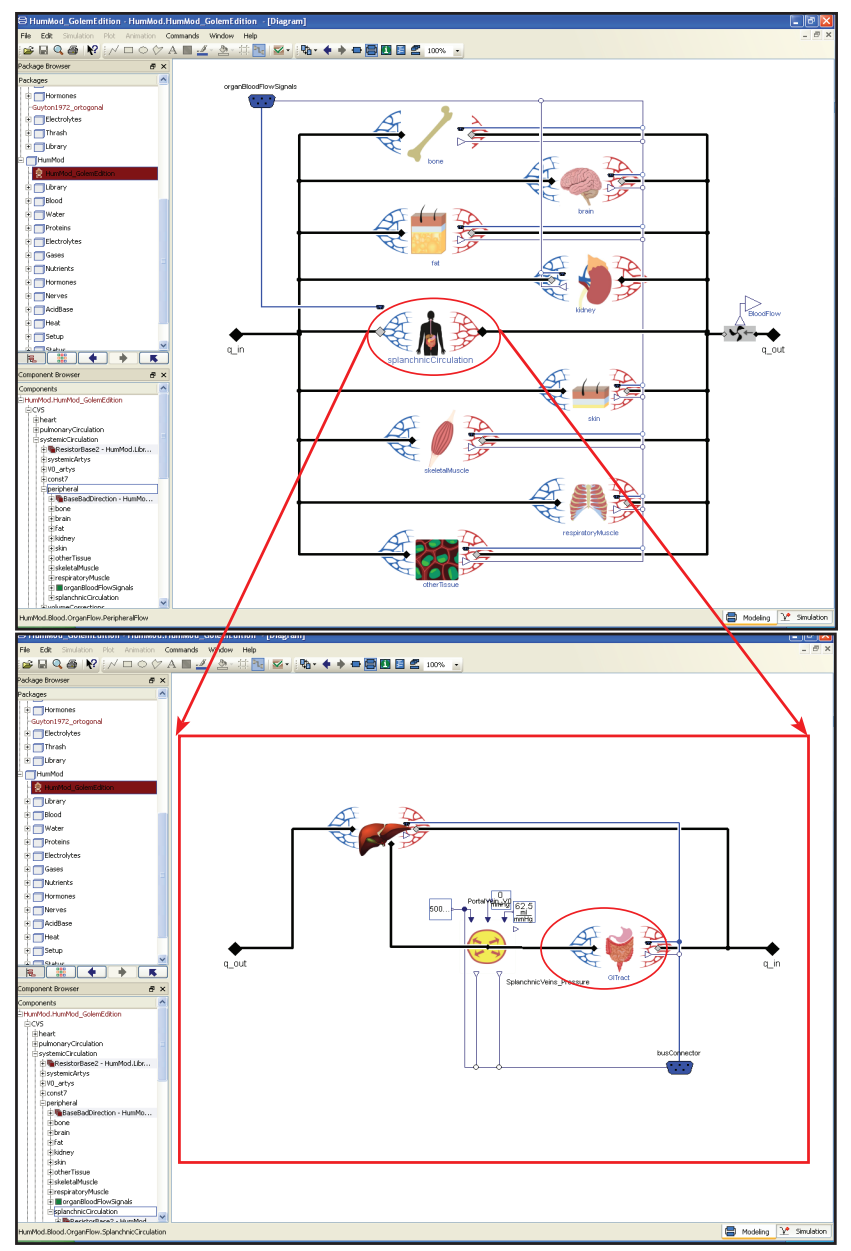

Figure 8. An illustration of a part of the source text of our HumMod implementation in Modelica. The source text resembles hierarchical physiological schemes. The content of the component of splanchnic circulation (from the upper figure) shows blood flow through the gastrointestinal tract component, the elastic compartment of the portal vein, and flow through the liver. 
We developed a special software tool called QHPView (J. Kofránek, Mateják, and Privitzer 2010) to create a clear visualization of the used mathematical relationships from the thousands of the source text files. This enabled us to orient ourselves in the large model.

The model reimplementation in Modelica resulted in a substantially better visualization of the model structure (see Fig. 8) and among other things, it also helped to discover some mistakes in the original American implementation of Hum Mod. We modified and expanded the model especially regarding the modelling of the transfer of blood gases and homeostasis of the inner environment, especially the acid-base balance (Jiri Kofranek, Matejak, and Privitzer 2011; Jirí Kofránek et al. 2013; Marek Mateják 2015; M. Mateják and Kofránek 2015).

Our version of HumMod called PHYSIOMODEL has been developed as an open source model. Source texts of the model (i.e. the equations, values of all constants, etc.) representing formalized expressions of physiological relationships are available to the public at http://www. physiomodel.org. The development of the integrative model of human physiology has also resulted in designing application libraries for the modelling of physiological and chemical systems in Modelica called "Physiolibrary" and "Chemical" (see http://www.physiolibrary.org) (Marek Mateják et al. 2014; Marek Mateják 2014; Marek Mateják et al. 2015; Matejak et al. 2015).

A more detailed description of the libraries and of our implementation of the integrative model of human physiology is the subject of a PhD thesis (Marek Mateják 2015).

\section{Importance of integrative models}

A relatively logical question emerges in a connection with the relatively demanding activities of developing integrated models - what can these models, created while exerting such great efforts, be used for?

\subsection{Understanding of the context}

The main benefit of these models consists in understanding how the human organism works as a whole, being a hierarchical system with complex regulations; how individual disturbances are manifested representing the bases of various diseases; and how an appropriate therapy is applied.

The reason why actually Guyton with his collaborators created the model cited in the introduction can also be given as an example (A. C. Guyton, Coleman, and Granger 1972). It was for the study of regulatory disorders resulting in high blood pressure, for the study of effects that control the heart pump activity, and for exploration of adaptive responses to a heart failure (A. C. Guyton, Granger, and Coleman 1971; A. C. Guyton 1981). The model has helped to understand the mechanisms of these actions.

In the past, when the physiologists focused only on the study of the dynamics of blood circulation, a simple mechanistic notion existed saying that high blood pressure was caused by an elevated peripheral resistance of blood vessels. Clinical findings in hypertonic patients corresponded to this notion - some of them actually did have increased peripheral resistance. However, we can ask why in some diseases associated with increased peripheral resistance (for example, hypothyroidism or in conditions after amputation of multiple limbs) the blood pressure is normal? Also, blood pressure remains unchanged in some diseases where peripheral resistance is decreased - for example, hyperthyroidism, beriberi, anaemia or arteriovenous shunts. It has shown, that exploration of regulation in the circulatory system alone is insufficient to explain these phenomena; we need to take into account also the regulation of volume and osmolarity of body fluids and the regulation of water and salts intake and output. Namely arterial blood pressure depends, among others, not only on peripheral vascular resistance but also on the contents of the vascular bloodstream, i.e. on the overall volume of circulating blood and also on the cardiac output. Blood pressure rises together with the volume of circulating blood. Kidneys promptly respond to this situation, excrete the excessive volume, and the blood pressure is adjusted. When the heart starts pumping more blood in a time unit - i.e. when the output increases, while peripheral resistance does not decrease at the same time, blood pressure rises, as well. On the other hand, the heart is a special pump that is controlled also by the pressure at its output - heart output changes also when pressure increases in large veins at the input of the atria of the heart. When the heart output remains increased in the long term, it gradually leads to a regulatory response in the periphery where peripheral resistance rises in order to reduce chronic hyperperfusion of internal organs. As shown by the research of Guyton using simulation models, the pathogenesis of the hypertension disease consists in disorders of these regulatory mechanisms - the kidneys are wrongly set to regulating a larger volume of circulating blood; increased contents of the bloodstream leads to an increase of the contents of large veins; this causes an increase in the pressure in large veins; and the increased pressure at the input of the atria of the heart causes increased heart output responded to in the periphery by increased peripheral resistance after some time (in order to reduce the hyperperfusion of peripheral organs), and thereby the increased blood pressure becomes fixed (A. C. Guyton, Hall, and Montani 1988).

In his models, Guyton also showed the mechanism of adaptive response to heart failure where, again, mechanisms related to circulatory and volume regulation are applied (J.-P. Montani and Van Vliet 2009). The 
results of these simulation studies have found their way to medical textbooks.

Guyton himself paid great attention to teaching of physicians and wrote a generally recognized textbook of physiology that provides a logical explanation of the mechanism of physiological regulatory actions. Guyton died in 2003 in a car accident, but his collaborators and students continue his work - they not only elaborated the original Guyton's model creating the above mentioned extensive model HumMod, but they also continue publishing his textbook complementedwith new knowledge - currently, the 13th edition of this textbook is available (Arthur C. Guyton and Hall 2015).

The extended integrated model has also found application in cosmic medicine. For example, the disciples of Guyton succeeded in using the model to explain why the adaptation to the gravitational force of the Earth after returning from an orbit takes about 5 times longer in female than male astronauts. Model simulations showed the cause of this phenomenon. In females, the centre of gravity is found lower than in males due to anatomical differences. The extracellular space is dewatered in the weightless condition and rewatered again after returning to the atmosphere - in females, the volume of fluids moved back from the blood to the interstitium is larger than in males due to the shifted centre of gravity, resulting in prolonged adaptation to the force of gravity - for details see (Summers et al. 2010).

Simulation games with an integrated model can also contribute to the guidelines for some procedures in acute medicine. For example, HumMod showed why (and for how long) it is important to preoxygenate the patient with $100 \%$ inhalatory oxygen before intubation (during anaesthesia) (this is a guideline for anaesthesia) - the patient namely does not breathe for a certain period of time during the intubation procedure. Furthermore, as shown by the model, it is needless to apply preventive hyperventilation after intubation and after connecting the patient to artificial pulmonary ventilation (which used to be the routine approach of some anaesthesiologists) - for details see (Lerant et al. 2015).

One of the body's most critical tasks is water homeostasis. Physical challenges to the body, including exercise and surgery, almost always coordinate with some change in water handling reflecting the changing needs of the body. The HumMod integrative mathematical model of human physiology was also validated against six different challenges to water homeostasis with special attention to the secretion of vasopressin and maintenance of electrolyte balance. HumMod successfully replicated the experimental results, remaining within 1 standard deviation of the experimental means in 138 of 161 measurements. Only three measurements lay outside of the second standard deviation. This validation suggests that
HumMod can be used to understand water homeostasis under a variety of conditions (W. A. Pruett, Clemmer, and Hester 2016).

As shown by the examples above, the use of integrated models can help to explain causal relationships of a number of physiological actions.

\subsection{Populations of virtual patients for clinical studies}

In order to explain the course of pathogenesis of various diseases and responses of people to administered therapy, it is important to ensure that the integrated model represents more than a kind of an average person. Sensitivity analysis can show how the changes in values of individual parameters are manifested in the overall behaviour of the model. For the purpose of studying individual responses, the integrated model representing a "normal" patient is used to create a population of models representing the population of various patients by variation of parameter values (approx. by $+/-10 \%$ ). Precisely this approach then makes its possible to observe individual variability of behaviour of the model and compare the same to individual variability of the population of real patients.

Thus, for example, in the study of individual responses to bleeding (Zhang, Pruett, and Hester 2015) the population of 395 patients was first created using this method. About $85 \%$ of the thus created population

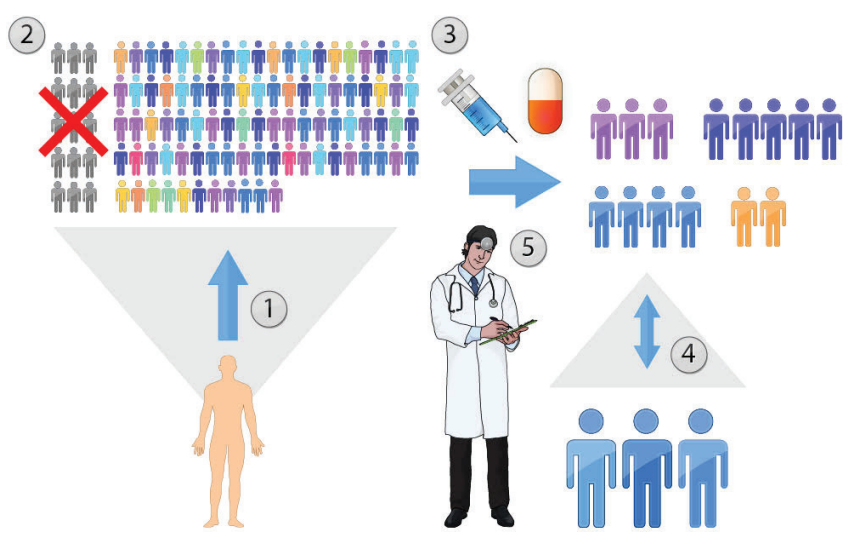

Figure 9. A possible way of using integrative models for interpretation of clinical study results. (1) A variation of parameter values is used to create a population of virtual patients. (2) Patients whose variable values exceed normal ranges are excluded from the thus created population of patients. (3) The remaining "healthy" heterogeneous population of virtual patients is used to perform the clinical experiment (simulated administration of pharmaceuticals). (4) The virtual patients are sorted in groups with similar responses to the virtual therapy. (5) For the given groups of virtual patients, we try to find matching groups of real patients with similar responses in the clinical study. Analysis of behaviour of the simulation model during the simulated therapy is used to seek explanations of individual differences in responses to the administered therapy. 
of virtual patients showed normal physiological values - and only $15 \%$ showed abnormal values, which were removed from the population. This is how a single integrated model of the "average" patient was used to create the population of models representing a set of individual (virtual) patients. And this heterogeneous population was then used for research aimed at revealing the causes of individual deviations in patient responses to a pathogenic noxa (bleeding in the given case) or to administered therapy. Results of the study (behaviour of virtual patients in haemorrhage) were then classified using cluster analysis in order to sort patients with similar behavioural patterns; these groups of virtual patients were then compared to similar behavioural patterns in real patients. Subsequently, qualitative analysis of the model behaviour could be done in order to find the causes of the individually different responses.

The analysis of sensitivity of parameters affecting the blood pressure value was performed similarly, resulting in the population of individual models with similar behavioural patterns based on an older model of Guyton (Moss et al. 2012).

It thus seems that the path leading to future application of integrative models in clinical situations (especially in clinical studies) consists in generating a population of models representing the population of virtual patients, subsequent modelling of the given pathology or effect of medications using this heterogeneous population of models, and sorting of the simulated virtual patients to groups according to similar responses. Based on comparison with a group of similarly responding patients of the clinical study, analyses of the model behaviour can reveal the causes of different responses in the patient groups to the given pathogenic noxa or to the therapy (see Fig. 9).

The pathology or the effect of the therapy in integrated models is usually modelled by changing some parameters that cause an appropriate (pathogenic or therapeutic) response.

As follows from simulation studies, the cause of differences of some individual responses need not be based on a difference in only one parameter - but in combined changes of several parameters.

Let us explain this problem using an illustrative example (Fig. 10) (P. Kohl et al. 2010). For a clearer idea, only the parametric state space of two parameters, P1 and $\mathrm{P} 2$ will be considered. The value of the hypothetic biological function is the axis $\mathrm{z}$ differentiated by its height and colour. We shall consider a patient whose biological profile is located at the position A. The required action (simulating the effect of the therapy or the effect of a "side effect of some other medication") consists in reducing the parameter P1 to its target value. A direct change of parameter P1 (the path from A to C) leads to a serious

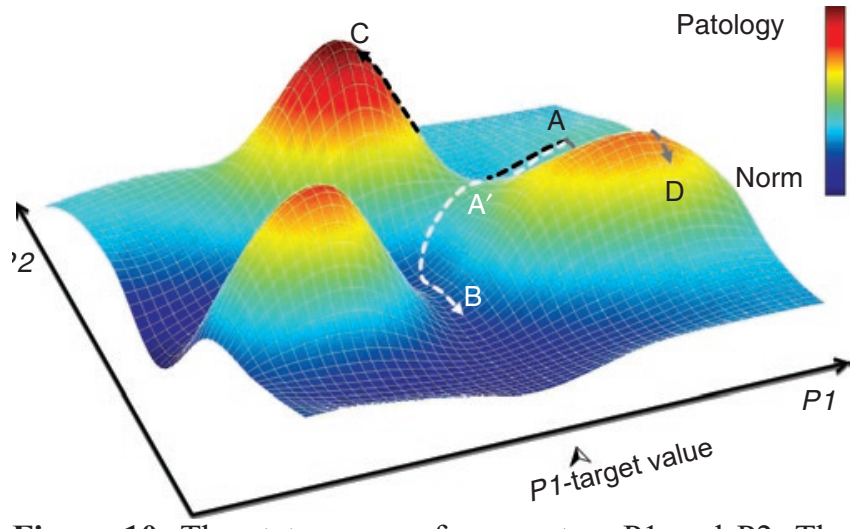

Figure 10. The state space of parameters P1 and P2. The vertical axis represents the size of the biological response with the given values of parameters $\mathrm{P} 1$ and $\mathrm{P} 2$. The purpose of a targeted intervention is to change the value of parameter P1 to its target value. No biological response is induced by a concurrent change of parameters $\mathrm{P} 1$ and P2 (path from A to B). The change of parameter P2 to the target value results in a biological effect (path from A to C). Similarly, if only parameter P2 changes (while parameter P1 remains unchanged), a biological response is induced. This illustrative example demonstrates that isolated changes of individual parameters may result in a biological effect while a covariant, concurrent change of two parameters may not cause any effect at all.

biological (pathological) response. The covariance of both parameters P1 and P2 (the path $\mathrm{A}-\mathrm{A}^{\prime}-\mathrm{B}$ ) makes it possible to move to the required level P1 without any harmful consequences. An isolated reduction of the parameter $\mathrm{P} 2$ in the same range as at the point $\mathrm{B}$ (without changing P1) would also be harmful; as can be seen intuitively, precisely the path of gradual modification of both parameters (P1 and P2) from A to B causes no biological response.

And vice versa - only a concurrent change of several parameters causes an unfavourable biological effect, while changes in individual parameters cause no adverse biological effect - and frequently, this is also the core of the robustness and ultrastability of physiological regulations that can be revealed precisely and only using integrative models.

Thus for example, variations of parameters of the HumMod model were used to monitor the sensitivity of blood pressure changes after a salt intake (W. Pruett, Husband, and Hester 2014). It was shown that no single parameter which would lead to an increased blood pressure after an increased salt intake existed - this is the core of high stability of physiological regulations. Only the change of several parameters resulted in a pathological response.

By comparing classified groups of virtual patients with the same behaviour and groups of real patients in clinical studies - and subsequent qualitative analysis of the courses of the modelled actions, the cause of individual deviations 
in response to an appropriate stimulus can be revealed no matter whether the stimulus is a pathological noxa or the effect of a medication.

This is why integrated models will also find their future application in clinical studies. The project of the European Union called "AVICENNA - A Strategy for in silico Clinical Trials" (see http://avicenna-isct.org), currently under preparation, will focus on the topic of using simulation models in clinical studies.

\subsection{Medical simulators}

Medical simulators represent another extensive field for the application of integrative models; similarly as flight simulators, medical simulators provide quite a new mode of teaching where students can train diagnostic and therapeutic tasks in virtual reality without any risk for the patient. In sophisticated medical simulators, students can also observe in detail the course of values of various quantities that are commonly not available for clinical examination in real patients, which supports deeper understanding of the pathophysiological core of the development of the clinical condition and its affection by therapeutic interventions.

The important thing is that unlike the real world, mistakes are reversible in virtual reality. When a flight simulator is used to train landing we can crash many times in a row, while in the real world an airplane crashes only once as a rule. In acute care medicine, diagnostic and therapeutic procedures can be trained on a virtual patient who can be brought back to life at any time. However, patients in the real life have no "reset" button and, as expressed by one harsh proverb, "the mistakes of rescuers are covered by soil".

Similarly as a sophisticated airplane model is the core of flight simulators, an integrative patient model is the key component of current top medical simulators (for example, in CAE Healthcare simulators - see http://www. caehealthcare.com).

\section{Development tools for integrative models}

Formerly, dynamic systems were often programmed using analogue computers, while later they were combined with a digital computer in the so-called hybrid computers. The program was created by connecting individual computing elements (integrators, summators ...) using connection cables. The computer processed analogue (continuous) electrical signals whose changes were responded to immediately, and therefore it remained a suitable tool for solving sets of differential equations of simulation models until the increasing power of digital computers removed this advantage of analogue solutions.

\subsection{Classical programming languages for the}

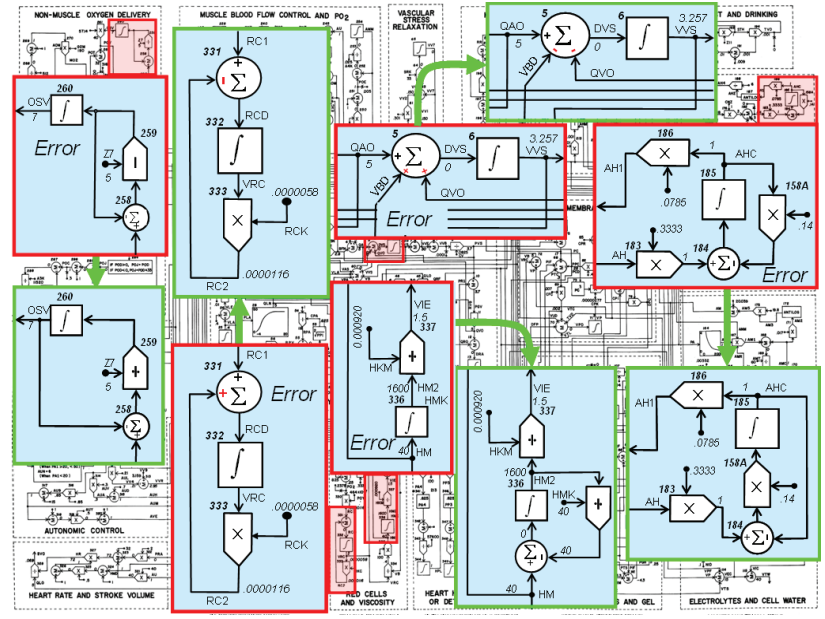

Figure 11. Mistakes in the Guyton's graphic scheme and their corrections.

\section{development of simulation models}

The era of analogue computers inspired also the graphic notation of Guyton used to write physiological models using a network of mutually connected computing blocks (integrators, summators, dividers, multipliers and function blocks). However, in 1972, at the time when the groundbreaking paper of Guyton (A. C. Guyton, Coleman, and Granger 1972) was published, models were implemented predominantly on digital computers using classical programming languages (e.g.Fortran, $\mathrm{C}, \mathrm{C}++$ etc.). The graphic scheme in the paper served only as an illustrative picture used to provide a compact description of the model structure. The model itself was programmed in the programming language Fortran for digital computers.

However, this scheme was not flawless (Jiří Kofranek and Rusz 2010) - some errors were apparent at first sight (for example, a wrongly connected integrator that would soon result in its overloading with an infinitely rising value due to the feedback), while others required a deeper analysis, understanding of the text of the article and knowledge of physiology (Fig. 11). Actually, these were easily detectable "graphic typing errors" (switched signs, shifted connectors) without any effect on the model functionality because the entire scheme was created only as an illustration and not as the source code of the model (programmed in Fortran). The picture itself was a part of the $\mathrm{PhD}$ thesis of a co-author of the Guyton's article, Thomas Coleman, and today, it can be find as a certain scientific relic in a display case of the Guyton's research centre at the University of Mississippi.

\subsection{Simulation chips in block-oriented languages}

At the beginning of the 1990s, specialized modelling tools emerged. These tools used computing blocks (very similar to those used by Guyton in his graphic notation); these blocks are connected on the computer screen using the mouse to create a simulation network. 


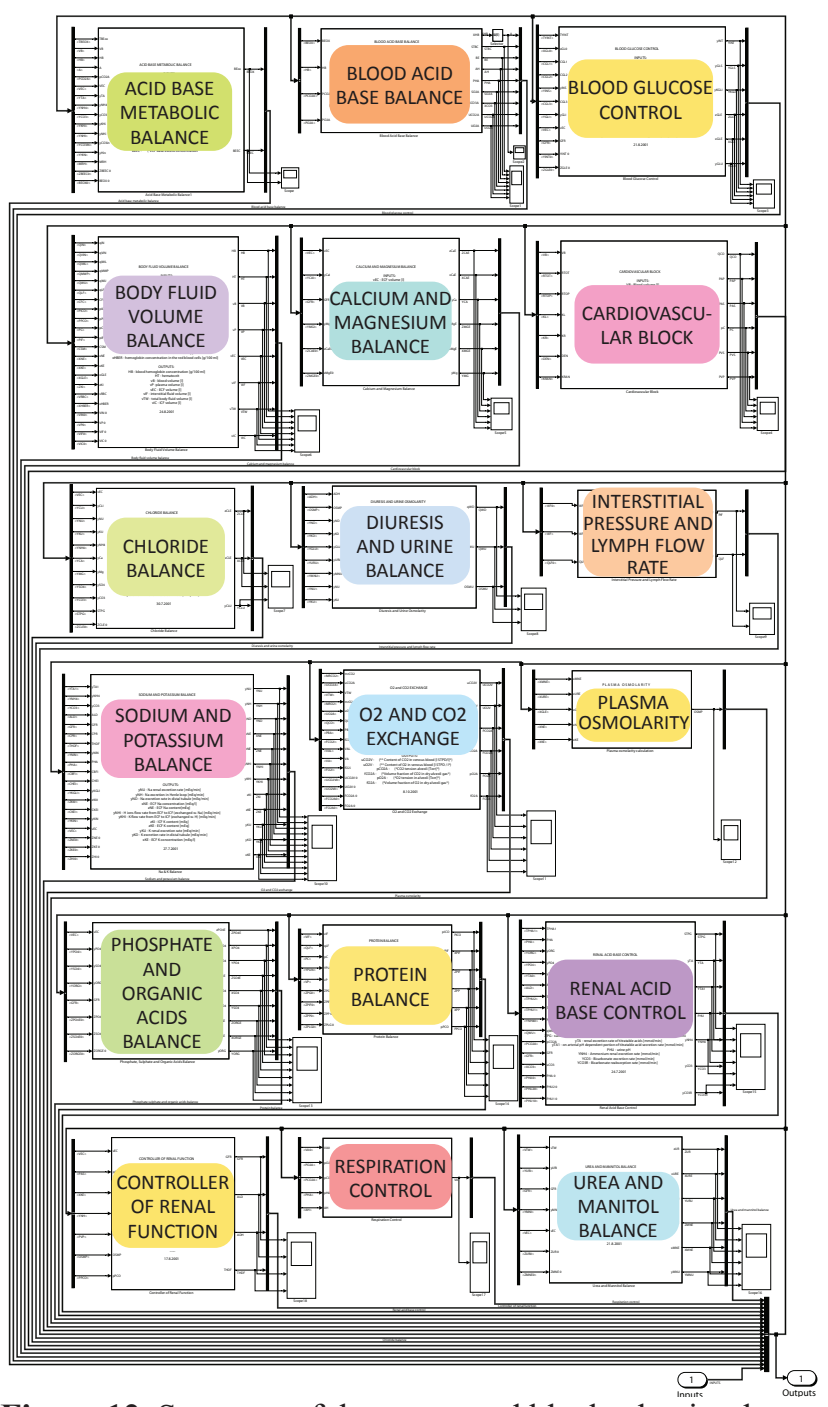

Figure 12. Structure of the connected blocks that implement the model for the Golem simulator in the block-oriented language Simulink. The inputs and outputs of 18 blocks modelling individual physiological subsystems are connected through a common bus.

These so-called block-oriented simulation languages utilize connected blocks. Signals "flow" through the connecting lines between individual blocks and transfer the values of individual variables from the output of one block to the inputs of other blocks. The inputted information is processed in the blocks to obtain the output information. The connections among individual blocks show how the values of individual variables are calculated - i.e. the algorithm of the computation.

Blocks can be grouped in individual subsystems that communicate with their surroundings through input and output "pins", thus representing certain "simulation chips". These subsystem blocks hide the simulation network structure from the user, similarly as electronic chips hide the connection structure of individual transistors and other electronic elements so that the user need not take care of the internal structure and of the computational algorithm used to obtain output variables from input variables. "Simulation chips" in block-oriented languages have a hierarchical structure - they may contain a network of mutually connected subsystem blocks of a lower hierarchical level. "Simulation chips" can be grouped in libraries and their individual instances can be created using the mouse; their inputs and outputs are connected using connecting lines, through which information (i.e. the values of variables) "flows". The entire complex model can be thus depicted as interconnected simulation blocks, while the structure of their connection provides clear information about what values are calculated and how.

This facilitates interdisciplinary cooperation in the development of integrative models where experimental physiologists do not have to explore in detail what mathematical relationships are hidden inside the connected subsystem blocks, and from the connections among individual subsystem blocks the physiologists can understand the model structure and verify the model behaviour in an appropriate simulation visualization environment of the block-oriented simulation language.

Block-oriented simulation languages provided a considerable simplification of implementation of simulation models. The most widely used block-oriented languages include, for example, Mathworks Simulink (http://www.mathworks.com/products/simulink) Visual Solution VisSim (http://www.vissim.com).

In the past, we used Simulink to create a freely available library of blocks for the modelling of physiological systems (http://www.physiome.cz/simchips), which also included the source code of an integrated model of physiological systems used as a source for our teaching simulator Golem (Fig. 12). The teaching simulator Golem was developed by us at the end of the 1990s and at the turn of the millennium it was intended for teaching the homeostasis of the inner environment in clinical physiology. The simulator was used at some national as well as foreign faculties of medicine (Jiří Kofránek et al. 2001).

\subsection{Disadvantages of block-oriented simulation languages}

Blocks in block-oriented languages have a hierarchical structure. On the lowest level, the blocks are created as a network of interconnected numeric blocks that use input values to calculate output values. The connections among the numeric blocks represent a solution of mathematical equations of the model designed so that output values are calculated from input values.

However, the connection of blocks in the network of relationships cannot be arbitrary. No algebraic loops may occur in the connected elements - i.e. cyclic structures where an input value brought to the input of a computing block depends in the same time step (through several 
intermediators) on the output value of the same block.

Development environments of block-oriented languages provide tools to avoid algebraic loops; however, their use often results in transformations that make the model structure less clear.

The main problem of block-oriented languages is that the simulation network composed of hierarchically connected blocks shows a graphic representation of a chain of transformations of input values to output values, meaning that when the model is designed, an exact computational algorithm must be defined from input to output values of the model.

The requirement of a fixed direction of connections from inputs to outputs means that the connections of the blocks reflect the computation procedure and not the very structure of the modelled reality.

For example, when the direction of the computation is reversed (by replacing inputs with outputs), the algorithm will be different although the model equations remain the same. Thus for example, in the model of an electrical RLC circuit (or its hydraulic analogy) it will make a difference if the voltage (pressure in the hydraulic domain) or (electrical or hydraulic) current is used as the input for the circuit although the electrical (hydraulic) scheme itself does not change. The Simulink network representing the computational process will be different.

In complex models, it is usually not simple to derive the computation causality (i.e. to derive the algorithm of computing output variables from input variables).

\subsection{Modelica - the best tool for development models of integrative physiology}

At the turn of the millennium, a completely new category

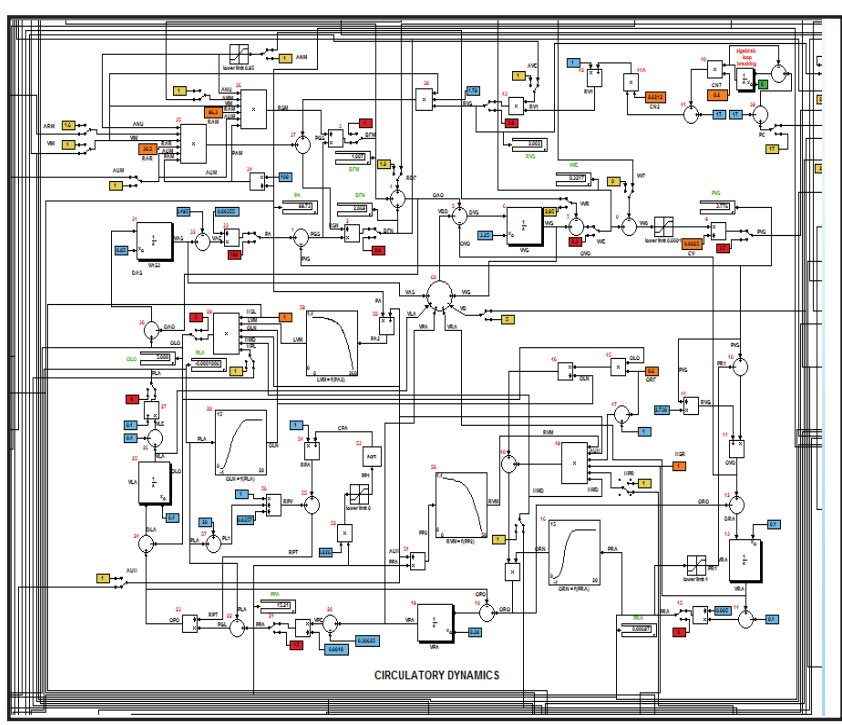

Figure 13. Circulatory dymamics - more detailed central structures of the Simulink implementation of Guyton's model, representing flows through aggregated parts of the circulatory system and the activity of the heart as a pump.. of modelling tools emerged, which makes it possible to leave the computation aside and describe directly equations in the modelling blocks. A special objectoriented equation-based language called Modelica was developed.

Modelica, originally developed as an academic project in cooperation with small development companies associated with Lund and Linköping universities, soon showed to be a very efficient tool for the modelling of complex models applied particularly in mechanical engineering, in the automobile and airline industries. The development of Modelica therefore gradually gained support of the commercial sector.

The speed at which the new simulation language Modelica spread in various industries and at which it was embraced by various commercial development environments is astonishing. Today, several commercial and non-commercial development tools exist that use this language (see https://www.modelica.org).

In Modelica, the connection of individual components results in the connection of sets of equations with each other. The component connections thus define the modelled reality instead of the computation process. The way of resolving the equations is thus "left up to the machines“.

Unlike block-oriented languages where the structure of connections among hierarchical blocks represents rather the computation process instead of the modelled reality, the structure of models in Modelica shows the structure of the modelled reality (see fig. 13 and 14). This is why even complex models are sufficiently transparent and comprehensible in Modelica (Kulhánek, Kofránek,

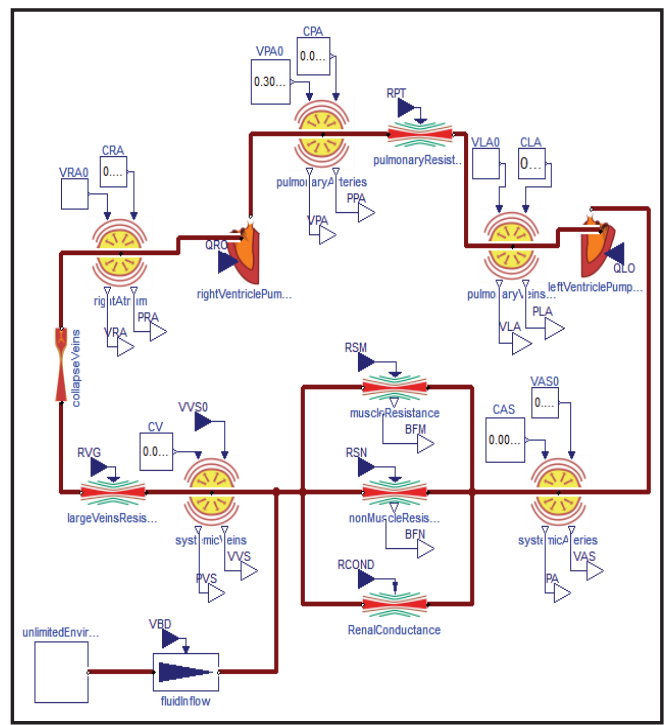

Figure 14. The same model structure as is shown in figure 13 implemented in Modelica. The structure of the model in Simulink corresponds to the structure of computational steps, while the Structure of Modelica model reflects the structure of the modeled physiological reality. 
and Mateják 2014).

This is very important precisely for the development of complex integrated models. The task of unifying and designing complex models faces another problem due to the complicatedness. Usually, only the authors are able to understand and use complex models. Modelica partially resolves this problem thanks to its characteristics and a complex model of human physiology designed in Modelica may lead to a wider use of the model in the scientific community. The source text of our integrated model of human physiology PHYSIOMODEL in Modelica (see http://www.physiomodel.org) resembles hierarchical physiological schemes (see Fig. 8). PHYSIOMODEL is an implementation of HumMod (modified and expanded, particularly in the field of acid-base balance and the transfer of blood gases) (Jiri Kofranek, Matejak, and Privitzer 2011; Jirí Kofránek et al. 2013; Marek Mateják 2015; M. Mateják and Kofránek 2015).

\section{Prospects of integrative models of human physiology}

\subsection{Prospects of sharing and publishing integrative models}

The development of integrative models in physiology exhibits an exclusively interdisciplinary nature. The team needs to have broad knowledge of physiology as well as knowledge of computer sciences, mathematics, the theory of control, and cybernetics. In addition, the team members of various professions must dispose of a considerable intersection of their knowledge.

This is also why there are not many scientific teams that develop large integrative models in physiology.

The developed integrative models should be comprehensible not only within the development team, but also externally - if a model is comprehensible only to its authors, it will hardly receive the necessary feedback and new impulses from specialized scientific community.

The issue of a suitable form of publishing the achieved results is also related to this issue. Reproducibility is the main attribute of any scientific result. Leaving aside certain acts of deception not discovered by reviewers, the principle of reproducibility is a key for the gradual discovery of the secrets of nature. This principle is often violated in the field of scientific publications related to biomedical models (both small and large). It is not always the fault of the authors - many times the reason is that a sign or an index is omitted in equations while the paper is prepared for printing, which causes a lot of problems to readers who seek not only to understand, but also to implement the described model.

In addition, in many cases biomedical models are as complex that the limited space for the article is sufficient only for fundamental equations of the model (and often not even all of them), while no space remains for more detailed information (initial values of state variables, values of all parameters, etc.), necessary to set up the model at a different department. Therefore the classical form of publications of models in journals is insufficient. A specialized article that describes a model should include, as a minimum, a digital (available through the Internet) appendix giving a detailed description of the model structure including the values of all parameters (preferably in the form designed in some modelling language), sufficient for the reader to be able to reproduce the model (and perhaps follow up in his or her own work). This solution has already been approached by a number of journals that publish specialized articles on computer models.

If the model is published in a modelling language that requires a commercial licence (for example, in MathWorks Matlab\&Simulink), a problem arises because the reader needs to have an appropriate licence to be able to run the given model in the licensed development environment.

Considerable efforts were thus developed in the international project PHYSIOME to create databases repositories of models that, besides storing the source text of the model in the defined format, offer publicly available tools for their simulation. Given that Modelica is a standardized language - and not a corporate proprietary product (such as MathWorks Matlab\&Simulink) and given that open source development tools exist today for this language (for example, OpenModelica - see https:// openmodelica.org) - Modelica seems to be a highly promising tool for publishing and sharing biomedical models.

So far, no other open source alternative besides Modelica exists that could be used for publishing extensive models. For example, Guyton's model version of 1992, implemented by Montani in C using the C-MODSIM environment (J. P. Montani, Adair, et al. 1989), is divided in the cellML repository in 22 modules in the open source cellML language. However, attempts at running these modules connected in one unit were not successful (https://models.cellml.org/exposure), while the Simulink version of the model works without problems (Mangourova, Ringwood, and Van Vliet 2011) (however, it requires the commercial environment of Matlab@ Simulink).

For the sake of completeness, we should note that theoretically, also the environment used to publish HumMod is an open source environment for implementation of large models - the source code of the model is saved in a number of XML files. This is sufficient for simple models; however, complex models are difficult to be understood by users - the reader can compare for themselves the HumMod model structure in the original form (http://hummod.org) and its implementation in 
Modelica (http://www.physiomodel.org).

Modelica thus seems to be a promising publication tool for extensive integrated models.

\subsection{Prospects of commercial application of integrative models}

The potential of commercial application especially in the two areas below will provide a powerful stimulus for further development of integrative models of human physiology:

- $\quad$ in medical teaching simulators;

- $\quad$ in the development of new therapeutic methods and in clinical testing of new pharmaceuticals.

Medical simulators provide a very efficient teaching aid. They enable the students to train basic examination and therapeutic techniques and also the process of decision-making in medicine. Sophisticated medical simulators utilize a robotized patient mannequin as the user interface. A model of interconnected physiological systems of the body is the core of modern medical simulators. Integrative physiology and integrated models of physiological systems thus become the technological know-how for the development of products with a high added value in the form of medical information and robotic knowledge that can find applications on the rapidly developing market.

Integrative models of human physiology will allow detailed monitoring of causal chains of application of various therapeutic or pathogenic stimuli, thus providing a wide potential for application of integrative models of human physiology especially in clinical testing of pharmaceuticals and in the development and testing of modern medical instruments (see Section 3.2).

The pressure of possible commercial applications leads to the fact that formalized descriptions of physiological regulations expressed as an integrated model often become carefully protected information, which limits the sharing of the results of scientific physiological research and undermines the possibilities of scientific cooperation.

\subsection{Prospects of combining commercial and academic development}

However, international cooperation and openness to sharing the results are the driving force of scientific development in today's globalized world. For example, as shown by experience, a community of users and developers as wide as possible is important for the development of complex software systems, thus a community that can provide feedback and ensure further innovations of a complex product through cooperative development, while subsequently, further entrepreneurial opportunities open up in the connection with this product - this is why such a great spreading of the development of projects with the open source code has been seen in recent years.
In order to ensure the development of complex integrated models in physiology, it will probably be suitable to seek such forms that will combine entrepreneurial opportunities and financing by the commercial sector with open scientific development.

One of the possibilities is to utilize a similar form in which the product OpenModelica has been developed in the open community (see https://openmodelica.org). The development of products is ensured by the consortium of 23 universities and 23 companies and institutions as well as a number of individual developers (Open Source Modelica Consortium - see https://openmodelica.org/ home/consortium). Research is financed using member contributions whose amount is determined based on the size of the company and based on the number of sold products in whose development OpenModelica licences have been used. OpenModelica has created a circle of a relatively large community of users as well as a high number of cooperating developers; the result is a functional open source product equivalent in terms of functionality to competitive expensive commercial implementations of Modelica such as Dymola from Dassault Systèmes), MapleSim from MapleSoft), Wolfram SystemModeler from Wolfram, etc. Commercial companies may use and further develop any part of the OpenModelica environment in their own commercial applications, also in the development of competitive commercial implementations of Modelica (this is why companies such as Wolfram Math Core or MapleSoft are also members of the consortium).

Perhaps a consortium of the academic community and commercial companies built on similar foundations called e.g. "Physiomodelica Open Source Consortium" could ensure further development of an integrated model of physiology in the future.

\section{Acknowledgements}

The authors appreciate the partial funding of this work by PRVOUK P/24/LF1 and FR Cesnet 551/2014. 


\section{References}

Amosov, N. M., B. L. Palec, B. T. Agapov, I. I. Jermakova, E. G. Ljabach, S. A. Packina, and V. P. Solovjev. 1977. Theoretical Research of Physiological Systems: Matematical Modeling (in Russian). Naukova Dumka.

Atkins, Gordon Leslie. 1969. Multicompartment Models for Biological Systems. Methuen London.

Bassingthwaighte, J. B. 2000. "Strategies for the Physiome Project.” Annals of Biomedical Engineering 28 (8). Springer: 1043-58.

Chen, Jian, Keqin Wu, William A. Pruett, and Robert L. Hester. 2013. "HumMod Browser: An Exploratory Visualization Tool for Model Validation of Whole-Body Physiology Simulation." In Eurographics Conference on Visualization (EuroVis)(short Paper). researchgate. net. https://www.researchgate.net/profile/Keqin_Wu2/ publication/303290077_HumMod_Browser_An_Exploratory_Visualization_Tool_for_Model_Validation_of_Whole-Body_Physiology_Simulation/ links/573f6ab108ae298602e8f3cf.pdf.

Coleman, T. G., and R. L. Summers. 1997. "Using Mathematical Models to Better Understand Integrative Physiology." Journal of Physiology and Biochemistry 53: 45-46.

Fontecave-Jallon, J., and S. R. Thomas. 2015. "Implementation of a Model of Bodily Fluids Regulation." Acta Biotheoretica 63 (3): 269-82.

Grodins, F. S., J. Buell, and A. J. Bart. 1967. "Mathematical Analysis and Digital Simulation of the Respiratory Control System.” Journal of Applied Physiology 22 (2). DTIC Document: $260-76$.

Guyton, A. C. 1981. "The Relationship of Cardiac Output and Arterial Pressure Control." Circulation 64 (6): 1079-88.

Guyton, A. C., T. G. Coleman, and H. J. Granger. 1972. "Circulation: Overall Regulation.” Annual Review of Physiology 34. annualreviews.org: 13-46.

Guyton, A. C., H. J. Granger, and T. G. Coleman. 1971. “Autoregulation of the Total Systemic Circulation and Its Relation to Control of Cardiac Output and Arterial Pressure." Circulation Research 28 (January): Suppl 1:93-97.

Guyton, A. C., J. E. Hall, and J. P. Montani. 1988. "Kidney Function and Hypertension.” Acta Physiologica Scandinavica. Supplementum 571: 163-73.

Guyton, A. C., R. D. Manning Jr, R. A. Norman Jr, J. P. Montani, T. E. Lohmeier, and J. E. Hall. 1986. "Current Concepts and Perspectives of Renal Volume Regulation in Relationship to Hypertension." Journal of Hypertension. Supplement: Official Journal of the International Society of Hypertension 4 (4): S49-56.

Guyton, A. C., and John E. Hall. 2015. Guyton and Hall Textbook of Medical Physiology. Elsevier Health Sciences.

Hester, R., A. Brown, L. Husband, and R. Iliescu. 2011. "HumMod: A Modeling Environment for the Simulation of Integrative Human Physiology." Frontiers in. journal.frontiersin.org. http://journal.frontiersin.org/article/10.3389/ fphys.2011.00012.

Hester, R. L., T. Coleman, and R. Summers. 2008. "A Multilevel Open Source Integrative Model of Human Physiology.” The FASEB Journal 22 (1 Supplement): 756.8-756.8.

Hester, R. L., R. Iliescu, R. Summers, and T. G. Coleman.
2011. "Systems Biology and Integrative Physiological Modelling." The Journal of Physiology 589 (Pt 5). Wiley Online Library: 1053-60.

Hodgkin, A. L., and A. F. Huxley. 1952. "A Quantitative Description of Membrane Current and Its Application to Conduction and Excitation in Nerve." The Journal of Physiology 117 (4). ncbi.nlm.nih.gov: 500-544.

Hunter, P. 2016. "The Virtual Physiological Human: The Physiome Project Aims to Develop Reproducible, Multiscale Models for Clinical Practice.” IEEE Pulse 7 (4). ieeexplore.ieee.org: $36-42$.

Hunter, P., J. Edmund, J. Crampin, and Poul M. F. Nielsen. 2008. "Bioinformatics, Multiscale Modeling and the IUPS Physiome Project." Briefings in Bioinformatics 9 (4). Oxford Univ Press: 333-43.

Hunter, P., P. Robbins, and D. Noble. 2002. "The IUPS Human Physiome Project." Pflugers Archiv: European Journal of Physiology 445 (1). Springer: 1-9.

Hunter, P. J., W. W. Li, A. D. McCulloch, and D. Noble. 2006. "Multiscale Modeling: Physiome Project Standards, Tools, and Databases." Computer 39 (11). ieexplore.ieee.org: 48-54.

Ikeda, N., F. M., M. Shirataka, and T. Sato. 1979. “A Model of Overall Regulation of Body Fluids." Annals of Biomedical Engineering 7 (2): 135-66.

Kofranek, J., M. Matejak, and P. Privitzer. 2011. "HummodLarge Scale Physiological Models in Modelica.” In Proceedings of the 8th International Modelica Conference; March 20th-22nd; Technical Univeristy; Dresden; Germany, 713-24. Linköping University Electronic Press.

Kofránek, J., M. Mateják, P. Privitzer, M. Tribula, T. Kulhánek, J. Šilar, and R. Pecinovský. 2013. "HumMod-Golem Edition: Large Scale Model of Integrative Physiology for Virtual Patient Simulators.” In Proceedings of the International Conference on Modeling, Simulation and Visualization Methods (MSV), 1. The Steering Committee of The World Congress in Computer Science, Computer Engineering and Applied Computing (WorldComp).

Kofranek, J., and J. Rusz. 2010. "Restoration of Guyton's Diagram for Regulation of the Circulation as a Basis for Quantitative Physiological Model Development.” Physiological Research / Academia Scientiarum Bohemoslovaca 59 (6). Institute of Physiology: 897.

Kofránek, J., L. D. Anh Vu, H. Snaselova, R. Kerekeš, and T. Velan. 2001. "GOLEM-Multimedia Simulator for Medical Education." Studies in Health Technology and Informatics, no. 2. IOS Press; 1999: 1042-46.

Kofránek, J., M. Mateják, and P. Privitzer. 2010. "Web Simulator Creation Technology.” MEFANET Report 3: 32-97.

Kohl, P., E. J. Crampin, T. A. Quinn, and D. Noble. 2010. "Systems Biology: An Approach." Clinical Pharmacology and Therapeutics 88 (1): 25-33.

Kohl, P., and D. Noble. 2009. "Systems Biology and the Virtual Physiological Human.” Molecular Systems Biology 5 (July): 292.

Kulhánek, T., J. Kofránek, and M. Mateják. 2014. “Modeling of Short-Term Mechanism of Arterial Pressure Control in the Cardiovascular System: Object-Oriented and Acausal Approach." Computers in Biology and Medicine 54 (November): 137-44. 
Lerant, A. A., R. L. Hester, T. G. Coleman, W. J. Phillips, J. D. Orledge, and W. B. Murray. 2015. "Preventing and Treating Hypoxia: Using a Physiology Simulator to Demonstrate the Value of Pre-Oxygenation and the Futility of Hyperventilation." International Journal of Medical Sciences 12 (8). ncbi.nlm.nih.gov: 625-32.

Mangourova, V., J. Ringwood, and B. Van Vliet. 2011. “Graphical Simulation Environments for Modelling and Simulation of Integrative Physiology." Computer Methods and Programs in Biomedicine 102 (3). Elsevier: 295-304.

Mateják, M.. 2014. "Physiology in Modelica." MEFANET Journal 2 (1). Facta Medica: 10-14.

Mateják, M. 2015. "Formalization of Integrative Physiology. Charles University in Prague.” Edited by Jiří Kofránek. Ph.D., Charles University. https://github.com/MarekMatejak/dissertation/blob/master/thesis.pdf.

Mateják, M-, F. Ježek, M. Tribula, and J. Kofránek. 2015. "Physiolibrary 2.3-An Intuitive Tool for Integrative Physiology." IFAC-PapersOnLine 48 (1). Elsevier: 699-700.

Mateják, M., T. Kulhánek, J. Šilar, P. Privitzer, F. Ježek, and J. Kofránek. 2014. "Physiolibrary-Modelica Library for Physiology." In Proceedings of the 10 Th International Modelica Conference; March 10-12; 2014; Lund; Sweden, 499-505. Linköping University Electronic Press.

Mateják, M., M. Tribula, F. Ježek, and J. Kofranek. 2015. "Free Modelica Library for Chemical and Electrochemical Processes." In Proceedings of the 11th International Modelica Conference, Versailles, France, September 21-23, 2015, 359-66. Linköping University Electronic Press.

Mateják, M., and J. Kofránek. 2015. "Physiomodel-an Integrative Physiology in Modelica." And Biology Society (EMBC), 2015 37th .... ieeexplore.ieee.org. http://ieeexplore.ieee.org/abstract/document/7318646/.

McCulloch, Warren S., and Walter Pitts. 1943. "A Logical Calculus of the Ideas Immanent in Nervous Activity." The Bulletin of Mathematical Biophysics 5 (4). Kluwer Academic Publishers: 115-33.

Milhorn, H. T. 1966. Application of Control Theory to Physiological Systems. W.B. Saunders.

Montani, J. P. and Bruce N. Van Vliet. 2009. "Understanding the Contribution of Guyton's Large Circulatory Model to Long-Term Control of Arterial Pressure." Experimental Physiology 94 (4). Wiley Online Library: 382-88.

Montani, J. P., T. H. Adair, R. L. Summers, T. G. Coleman, and A. C. Guyton. 1989. "A Simulation Support System for Solving Large Physiological Models on Microcomputers." International Journal of Bio-Medical Computing 24 (1): 41-54.

Montani, J. P., H. L. Mizelle, T. H. Adair, and A. C. Guyton. 1989. "Regulation of Cardiac Output during AldosteroneInduced Hypertension.” Journal of Hypertension. Supplement: Official Journal of the International Society of Hypertension 7 (6): S206-7.

Moss, R., T. Grosse, I. Marchant, N. Lassau, F. Gueyffier, and S. R. Thomas. 2012. "Virtual Patients and Sensitivity Analysis of the Guyton Model of Blood Pressure Regulation: Towards Individualized Models of WholeBody Physiology.” PLoS Computational Biology 8 (6): e1002571.

Omholt, S. W., and P. J. Hunter. 2016. "The Human Physiome:
A Necessary Key for the Creative Destruction of Medicine.” Interface Focus 6 (2). Royal Society: 20160003.

Pitts, W., and W. S. McCulloch. 1947. "How We Know Universals; the Perception of Auditory and Visual Forms." The Bulletin of Mathematical Biophysics 9 (3). Springer: 127-47.

Potůček, J., M. Hájek, V. Brodan, and E. Kuhn. 1977. "The Method of Estimating Biological System Parameters on Hybrid Computer.” Kybernetika 13 (2). Institute of Information Theory and Automation AS CR: 153-64.

Pruett, W. Andrew, John S. Clemmer, and Robert L. Hester. 2016. "Validation of an Integrative Mathematical Model of Dehydration and Rehydration in Virtual Humans." Physiological Reports 4 (22). doi:10.14814/phy2.13015.

Pruett, W., L. Husband, and R. Hester. 2014. "Understanding Variation in Salt Sensitivity in HumMod, a Human Physiological Simulator (857.11)." The FASEB Journal 28 (1 Supplement). http://www.fasebj.org/content/28/1_Supplement/857.11.abstract.

Sheppard, C. W. 1948. "The Theory of the Study of Transfers within a Multi-Compartment System Using Isotopic Tracers.” Journal of Applied Physics 19 (1). AIP: 70-76.

Shim, E. B, Ch. H. Leem, Y. Abe, and A. Noma. 2006. "A New Multi-Scale Simulation Model of the Circulation: From Cells to System." Philosophical Transactions. Series A, Mathematical, Physical, and Engineering Sciences 364 (1843): 1483-1500.

Summers, R. L., S. Platts, J. G. Myers, and T. G. Coleman. 2010. "Theoretical Analysis of the Mechanisms of a Gender Differentiation in the Propensity for Orthostatic Intolerance after Spaceflight.” Theoretical Biology \& Medical Modelling 7 (March): 8.

Thomas, S. R., P. Baconnier, J. Fontecave, J. P. Françoise, F. Guillaud, P. Hannaert, A. Hernández, et al. 2008. "SAPHIR: A Physiome Core Model of Body Fluid Homeostasis and Blood Pressure Regulation." Philosophical Transactions. Series A, Mathematical, Physical, and Engineering Sciences 366 (1878). rsta.royalsocietypublishing. org: 3175-97.

Von Bertalanffy, L. 1973. General Systems Theory. George Braziller Inc., New York.

White, R. J., and J. C. McPhee. 2007. "The Digital Astronaut: An Integrated Modeling and Database System for Space Biomedical Research and Operations.” Acta Astronautica 60 (4-7). Elsevier: 273-80.

Wu, K., J. Chen, W. A. Pruett, and R. L. Hester. 2013. "Hummod Browser: An Exploratory Visualization Tool for the Analysis of Whole-Body Physiology Simulation Data." In 2013 IEEE Symposium on Biological Data Visualization (BioVis), 97-104. ieeexplore.ieee.org.

Xu, L., J. Lyle, Y. Wu, Z Pan, M. Zhang, D. H. Laidlaw, R. L. Hester, and J. Chen. 2011. "HumMod Explorer: A MultiScale Time-Varying Human Modeling Navigator.” In SIGGRAPH Asia 2011 Posters, 28:1-28:1. SA '11. New York, NY, USA: ACM.

Zhang, S., W. A. Pruett, and R. Hester. 2015. "Visualization and Classification of Physiological Failure Modes in Ensemble Hemorrhage Simulation.” In SPIE/IS\&T Electronic Imaging, 939700 - $939700-8$. International Society for Optics and Photonics. 